\title{
Clinicopathological characteristics of patients with synchronous primary endometrial and ovarian cancers: A review of 43 cases
}

\author{
YUANTAO LIU, JUN LI, HONGYAN JIN, YING LU and XIN LU \\ Department of Gynecology, Obstetrics and Gynecology Hospital of Fudan University, Shanghai 200011, P.R. China
}

Received February 18, 2012; Accepted September 13, 2012

DOI: 10.3892/ol.2012.943

\begin{abstract}
Synchronous primary endometrial and ovarian cancers are uncommon. The purpose of this study was to evaluate the clinicopathological characteristics, treatment and prognosis of synchronous primary endometrial and ovarian cancers. The clinicopathological characteristics of 43 patients with synchronous primary endometrial and ovarian cancers in the Obstetrics and Gynecology Hospital of Fudan University between 1999 and 2009 were retrospectively reviewed. Our results revealed that the median age at the time of diagnosis was 51 years (range, 29-71). The common presenting symptoms were abnormal uterine bleeding (AUB, 65.12\%), abdominal mass $(25.58 \%)$, abdominal pain and abdominal fullness (39.53\%). An elevated CA125 level was observed in the majority of patients $(n=20,76.9 \%)$. Endometrioid type accounted for $60.47 \%$ of uterine carcinomas and different pathological types, including serous adenocarcinoma, clear cell carcinoma, adenosquamous and acanthoadenocarcinoma, were also identified in synchronous primary endometrial and ovarian cancers. All patients underwent surgical intervention (hysterectomy and bilateral salpingo-oophorectomy with pelvic lymphadenectomy or debulking surgery). The 5-year survival rate was $86.05 \%$ and nine patients had recurrence (20.93\%). The early stage group (FIGO stages I and II) had more favorable prognosis than the advanced stage group (FIGO stages III and IV; $\mathrm{P}<0.05$ ). In conclusion, synchronous primary endometrial and ovarian cancers are different from either primary endometrial carcinoma or ovarian cancer and are usually identified at early stages with a good prognosis.
\end{abstract}

\section{Introduction}

Synchronous primary endometrial and ovarian cancers coexist in approximately $10 \%$ of all females with ovarian cancer and

Correspondence to: Dr Xin Lu, Department of Gynecology, Obstetrics and Gynecology Hospital of Fudan University, No. 419 Fangxie Road, Shanghai 200011, P.R. China

E-mail: xinlu98@yahoo.com

Key words: synchronous primary cancers, endometrial cancer, ovarian cancer, prognosis in $5 \%$ of all females with endometrial cancer. These cancers are uncommon and often misdiagnosed as FIGO stage III of endometrial cancer or FIGO stage II of ovarian cancer (1). Several molecular analyses, including immunohistochemistry, DNA flow cytometry and gene mutation analysis, have been adapted in order to elucidate more definitely the dilemma of diagnosis; however, to date there is no consensus on the most appropriate method $(2,3)$. Previous studies have suggested that patients diagnosed with synchronous primary cancers have a better overall prognosis than those classified as single organ disease with metastasis. A Gynecologic Oncology Group (GOG) study reported that 74 patients with simultaneously detected endometrial and ovarian cancer limited to the pelvis without distinction between independent primary sites and metastasis had a 5-year survival rate of $86 \%$ and a 10 -year survival rate of $80 \%$ (4).

In the present study, 43 patients with synchronous primary endometrial and ovarian cancers diagnosed and treated at the Obstetrics and Gynecology Hospital of Fudan University (Shanghai, China) between 1999 and 2009 were retrospectively reviewed.

\section{Patients and methods}

Patient characteristics. Between 1999 and 2009, the medical records and pathological reports in the Obstetrics and Gynecology Hospital of Fudan University (Shanghai, China) database of 43 patients were retrospectively reviewed. The pathological specimens were reviewed and diagnosed by two gynecological pathologists according to the criteria described by Young and Scully (5): i) histological dissimilarity of the tumors; ii) no or only superficial myometrial invasion of endometrial tumor; iii) no vascular space invasion of endometrial tumor; iv) atypical endometrial hyperplasia additionally present; v) absence of other evidence of spread of endometrial tumor; vi) ovarian unilateral tumor (80-90\% of cases); vii) ovarian tumor located in parenchyma; viii) no vascular space invasion, surface implants or predominant hilarlocation in ovary; ix) absence of other evidence of spread of ovarian tumor; $x$ ) ovarian endometriosis present; $x i$ ) different ploidy of DNA indices, if aneuploid, of the tumors; and xii) dissimilar molecular genetic or karyotypic abnormalities in the tumors.

All the patients were followed up for at least 12 months. The clinicopathological data included age at diagnosis, presenting symptoms, body mass index (BMI), parity, 
personal and family history, assisted examination, treatment, histology, grade and recurrence. Patients were considered lost to follow-up when the duration of follow-up was $<12$ months following surgery (if no recurrence had occurred within that period).

The study was approved by the ethics committee of Fudan University, Shanghai, China. Written informed patient consent was obtained from the patient.

Statistical analysis. The data were analyzed using SPSS 13.0 software. Recurrence-free interval rates were determined using the Kaplan-Meier method. Recurrence-free intervals were calculated even for patients who had relapse. The log-rank test was used to test differences in survival within variables. $\mathrm{P}<0.05$ was considered to indicate a statistically significant result.

\section{Results}

Age and BMI. The median age of the 43 patients at the time of diagnosis was 51 years (range, 29-71). Two (4.7\%) patients were aged $<40$ years and $18(41.9 \%)$ patients were aged $\leq 50$ years. A total of 17 patients were menopausal (39.5\%), 8 patients were nulliparous (18.6\%), the median BMI was $23.44 \mathrm{~kg} / \mathrm{m}^{2}$ (range, 15.79-36.33), 7 (16.3\%) of the 43 patients were obese (BMI $>28$ ). One patient received hormone replacement treatment prior to admission to hospital. Data shown in Table I.

Chief complaint and symptoms. The most common presenting symptoms were abnormal uterine bleeding (AUB; $n=28$; $65.12 \%)$, abdominal pain and abdominal fullness $(n=17$; $39.53 \%)$ and abdominal mass $(\mathrm{n}=11 ; 25.58 \%)$; only one case exhibited vaginal discharge $(\mathrm{n}=1 ; 2.3 \%)$. Data shown in Table I.

Laboratory findings. The CA125 level was assessed in 26 patients, 20 of whom had an elevated CA-125 level (76.92\%), the median CA125 was $161.7 \mathrm{U} / \mathrm{ml}$. Eight patients had $>500 \mathrm{U} / \mathrm{ml}$ (30.77\%) Data shown in Table I..

Assisted examination. Ultrasonography is the most common radiographic test to evaluate adnexal masses and endometrial thickening. A total of 40 patients underwent pelvic ultrasound examination, the results of which revealed that 34 patients had an abdominal mass (85\%), 17 patients had uterine enlargement (42.5\%), 9 patients had endometrial thickening (22.5\%), 17 patients were found to have a mass occupation in the uterine cavity (42.5\%) and 13 patients had pelvic hydrops (32.5\%). Data shown in Table I.

Pathological specimens from 25 patients were obtained by diagnostic curettage, and of these 23 patients had endometrial carcinoma and 2 had endometrial complex hyperplasia. One of the patient's ascites smear was positive prior to surgery.

\section{Pathological findings}

Clinical stage. Most patients were diagnosed as early stage (stage I and II) in both uterus and the ovary. According to FIGO stage, 27 cases were endometrial cancer stage I (62.8\%), 9 cases were endometrial cancer stage II (20.9\%) and 7 cases were endometrial cancer stage III (16.3\%). Of the ovarian cancers, 27 cases were stage I (62.8\%), 4 cases were stage
Table I. Characteristics of the patients with synchronous primary endometrial and ovarian cancers.

\begin{tabular}{lcc}
\hline Characteristic & Number & $\%$ \\
\hline Symptoms (n=43) & & \\
Abnormal uterine bleeding & 28 & 65.12 \\
Abdominal pain & 17 & 39.53 \\
Abdominal mass & 11 & 25.58 \\
Vaginal discharge & 1 & 2.3 \\
CA125 (n=26) & & \\
Normal & 6 & 23.08 \\
$<500$ U/ml & 12 & 46.15 \\
$>500$ U/ml & 8 & 30.77 \\
Ultrasound examination (n=40) & & \\
Abdominal mass & 34 & 85.0 \\
Uterine enlargement & 17 & 42.5 \\
Endometrial thickening & 9 & 22.5 \\
Mass in uterine cavity & 17 & 42.5 \\
Pelvic hydrops & 13 & 32.5 \\
D and C (n=25) & & \\
Endometrial carcinoma & 23 & 92.0 \\
Complex hyperplasia & 2 & 8.0 \\
\hline
\end{tabular}

$\mathrm{D}$ and $\mathrm{C}$, dilation and curettage.

Table II. Histological characteristics of 43 patients.

\begin{tabular}{lccrcc}
\hline & \multicolumn{2}{c}{ Endometrial cancer } & & \multicolumn{2}{c}{ Ovarian cancer } \\
\cline { 2 - 3 } \cline { 6 - 7 } FIGO stage & Number & $\%$ & & Number & $\%$ \\
\hline I & 27 & 62.8 & & 27 & 62.8 \\
II & 9 & 20.9 & & 9.3 \\
III & 7 & 16.3 & & 12 & 27.9 \\
IV & 0 & 0 & & 0 & 0 \\
\hline
\end{tabular}

II (9.3\%) and 12 cases were stage III (27.9\%). Data shown in Table II.

Pathological type. Histological characteristics of the 43 cases are shown in Table III. Endometrioid cancer $(n=26,60.47 \%)$ was the main pathological type in uterine carcinoma and the other pathological types included serous adenocarcinoma, clear cell carcinoma, adenosquamous and acanthoadenocarcinoma. Ovarian cancer mostly occurred unilaterally $(\mathrm{n}=32 ; 74.4 \%)$, however 11 cases $(25.58 \%)$ had bilateral involvement of the ovary. The majority of pathological types were endometrioid adenocarcinoma and serous adenocarcinoma. In the present study, we noted specific pathological types of endometrial and ovarian cancers, such as endometrioid polypus canceration in the uterus with endometrioid carcinoma in the left ovary and carcinosarcoma in the right side. None of the 43 patients had vascular invasion or lymph node metastasis. 
Table III. Different pathological characteristics in synchronous primary endometrial and ovarian cancers.

Ovarian cancer

\begin{tabular}{lccccc}
\cline { 2 - 5 } Endometrial cancer & Endometrioid & Serous adenocarcinoma & Clear cell & Adenosquamous & Other \\
\hline Endometrioid & 12 & 1 & 1 & 2 & 9 \\
Serous adenocarcinoma & 0 & 7 & 1 & 0 & 0 \\
Clear cell & 0 & 0 & 0 & 0 & 1 \\
Adenosquamous & 0 & 0 & 0 & 0 & 1 \\
Other & 2 & 0 & 1 & 0 & 2
\end{tabular}

Table IV. Adjuvant therapy of the synchronous primary endometrial and ovarian cancers $(n=43)$.

\begin{tabular}{lcc}
\hline Adjuvant therapy & Number & $\%$ \\
\hline Neoadjuvant chemotherapy & 4 & 9.3 \\
Chemotherapy & 38 & 88.4 \\
Chemotherapy and radiotherapy & 3 & 6.98 \\
\hline
\end{tabular}

A

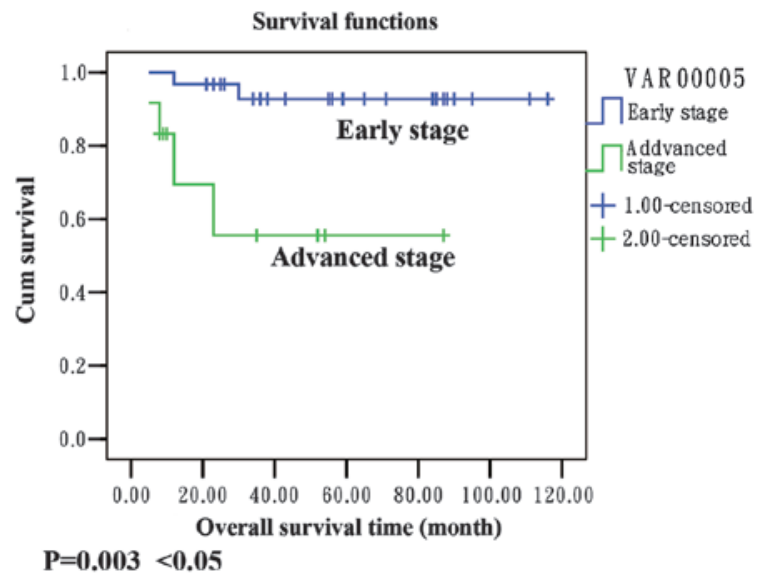

B

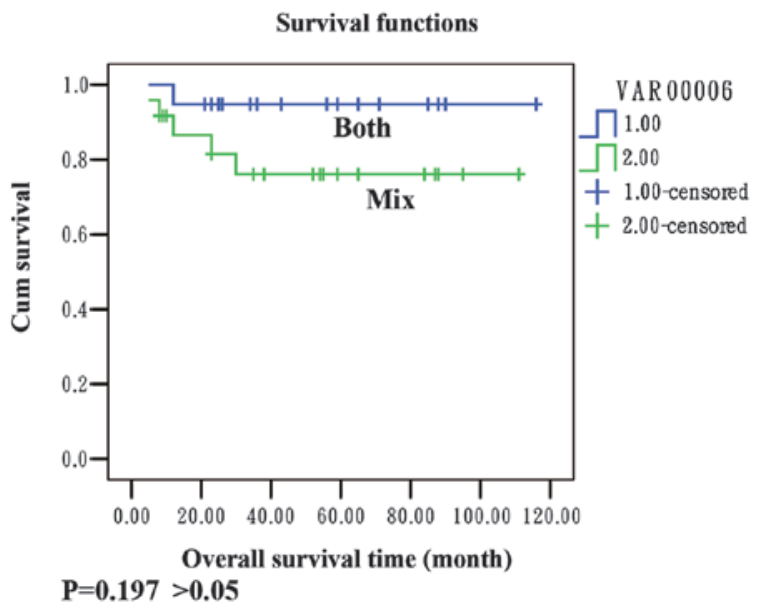

Figure 1. Overall survival and recurrence-free interval curves in patients who were followed up. (A) Survival analysis demonstrates a better survival in patients at early stage than those at advanced stage. (B) Patients with endometrioid endometrial cancer and endometrioid ovarian cancer had a better survival than patients with mixed pathological types.

\section{Treatment}

Surgical treatment. All patients initially underwent surgery in our hospital. Of the 43 patients, 6 had hysterectomy and bilateral salpingo-oophorectomy and the remaining 37 patients had total hysterectomy, bilateral salpingo-oophoretomy, omentectomy with appendectomy and pelvic lymph node dissection.

Adjuvant therapy. A total of 38 patients received platinbased adjuvant chemotherapy, 3 patients received both adjuvant chemotherapy and radiotherapy and 4 patients received neoadjuvant chemotherapy. Data shown in Table IV.

Recurrence and prognosis. Nine patients had recurrence (20.93\%). The median time to recurrence was 10 months (range, 5-30). The five-year survival rate of the patients was $86.05 \%$. Data shown in Fig. 1.

\section{Discussion}

Synchronous primary endometrial and ovarian cancers coexist in approximately $10 \%$ of all females with ovarian cancer and in $5 \%$ of all females with endometrial cancer. These cancers are uncommon and often misdiagnosed as FIGO stage III of endometrial cancer or FIGO stage II of ovarian cancer (1). In our study, the incidence was $3.08 \%$ in patients with endometrial cancer and $3.18 \%$ in patients with ovarian cancer. Our study was conducted in a single institution rather than multicenter analysis, the study only included cases with confirmed diagnosis of synchronous tumors and other conditions, such as primary endometrial cancer with ovarian metastasis and primary ovarian cancer with endometrial metastasis, were excluded.

Synchronous primary endometrial and ovarian cancers are unlike endometrial or ovarian cancer alone (6). Pathologists have listed histological criteria to evaluate these tumors. Molecular profiling in synchronous endometrioid and ovarian cancers may aid in determining a differential diagnosis. Halperin et al reported that $62.5 \%$ of synchronous primary endometrial and ovarian cancers can be classified by detection of ER and PR content and that $31.3 \%$ of synchronous primary endometrial and ovarian cancers can be identified by detecting Bcl-2 (6).

The median age of patients with synchronous primary endometrial and ovarian cancers was 50 years, and the median age of those with endometrial cancer or ovarian cancer was 60 years, so the incidence age of primary carcinoma was lower. Previous studies have also reported a younger median 
age in patients with synchronous primary endometrial and ovarian cancers (7). In a prospective series of 74 patients with simultaneously detected endometrial and ovarian cancers, the GOG reported a median age of 49 years. Synchronous primary endometrial and ovarian cancer patients are younger than those who develop endometrial or ovarian cancer alone. In our study, the median age of the patients at the time of diagnosis was 51 years (range, 29-71).

Obesity is a well-known risk factor for the development of endometrial cancer. In the study by Soliman et al, 36\% of patients (17/47) in the endometrioid/endometrioid group, $30 \%$ of patients (3/10) in the endometrioid/serous group and $40 \%$ of patients $(2 / 5)$ in the endometrioid/clear cell group were obese (BMI >30) (7). Nishimura et al reported that the mean BMI of Japanese females with synchronous primary endometrial and ovarian cancers was $22.6 \pm 3.4 \mathrm{~kg} / \mathrm{m}^{2}$ (range, 16-31) (8), but the authors did not report the obesity rates. In the present study, the median BMI was $23.44 \mathrm{~kg} / \mathrm{m}^{2}$ (range, 15.79-36.33), only $7(16.3 \%)$ of the 43 patients were obese (BMI >28). Asian ethnicity may be correlated with a lower obesity rate.

The clinical symptom and sign of synchronous primary endometrial and ovarian cancers are similar to the independent endometrial and ovarian cancers. AUB and abdominal mass were the main symptoms. In accordance with the results described in the literature, the common presenting symptoms in the present study were AUB (65.12\%), abdominal mass (25.58\%), abdominal pain and abdominal fullness (39.53\%).

Surgical treatment is the main treatment for endometrial and ovarian cancers. Synchronous primary endometrial and ovarian cancers are often misdiagnosed as FIGO stage III of endometrial cancer or FIGO stage II of ovarian cancer in early years, hence patients are often over-treated. Although cancer of both uterine body and that of the ovary occurs simultanenously, pathological changes are mainly of early stage. The scope of general surgery included hysterectomy, bilateral salpingo-oophorectomy, omentectomy and appendectomy. Pelvic lymph node dissection depended on the pathological findings $(9,10)$. The treatment of patients with ovarian cancer is generally based on chemotherapy with paclitaxel plus cisplatin (TP) regimen or cisplatin + doxorubicin + cyclophosphamide (PAC) regimen and the length of treatment ranges from 2 to 10 cycles, generally 3 to 6 cycles; however, patients with stage I or II grade 1 ovarian cancer do not require chemotherapy. In 1982, Eifel proposed that patients who have the following risk factors of endometrial cancer should receive adjuvant radiotherapy: i) pathological type of papillary serous adenocarcinoma or adenosquamous; ii) tumor differentiation as G2, G3 grade; iii) deep myometrial invasion.

However, the adjuvant treatment for these patients is controversial. In our study, surgical treatment was used for all patients; 6 patients underwent hysterectomy and bilateral salpingo-oophorectomy and the remaining 37 patients had total hysterectomy, bilateral salpingo-oophoretomy, omentectomy, appendectomy and pelvic lymph node dissection. The majority of patients received postoperative platinumbased chemotherapy. A total of 38 patients received adjuvant chemotherapy, 3 patients received both adjuvant chemotherapy and radiotherapy and 4 patients received neoadjuvant chemotherapy. It is difficult to evaluate the prognosis of these patients without consideration of the impact of adjuvant chemotherapy.

Our results showed that the mean survival in the group with early stage (I and II) disease was 109 months and the survival rate was $93.5 \%$; the mean survival was 54 months in the group with advanced stage (III/IV) disease and the survival rate was $63.7 \%$. There was a statistically significant difference between the groups $(\mathrm{P}=0.003<0.05$; Fig. $1 \mathrm{~A})$. The survival rate in the group of endometrioid carcinoma in the endometrium and ovaries was $94.1 \%$, the survival rate in the group with mixed pathological type was $80.8 \%$ and the result was not significant $(\mathrm{P}=0.197>0.05$; Fig. $1 \mathrm{~B})$. Ayhan et al concluded that the stage of ovarian cancer and grade of endometrial cancer are important prognostic factors (4). Our results indicated that the stage had a greater influence on the survival than the histology. We suggest that the advanced stage has a detrimental influence on survival and was a poor prognostic predicator in synchronous primary endometrial and ovarian cancers.

\section{References}

1. Zaino R, Whitney C, Brady MF, et al: Simultaneously detected endometrial and ovarian carcinomas - a prospective clinicopathologic study of 74 cases: a gynecologic oncology group study. Gynecol Oncol 83: 355-362, 2001.

2. Kaneki E, Oda Y, Ohishi Y, et al: Frequent microsatellite instability in synchronous ovarian and endometrial adenocarcinoma and its usefulness for differential diagnosis. Hum Pathol 35: 1484-1493, 2004.

3. Ricci R, Komminoth P, Bannwart F, et al: PTEN as a molecular marker to distinguish metastatic from primary synchronous endometrioid carcinomas of the ovary and uterus. Diagn Mol Pathol 12: 71-78, 2003.

4. Ayhan A, Guvenal T, Coskun F, et al: Survival and prognostic factors in patients with synchronous ovarian and endometrial cancers and endometrial cancer mestastatic to the ovaries. Eur J Gynaecol Oncol 24: 171-174, 2003.

5. Young RH and Scully RE: Metastasic tumors of the ovary. In: Blaustein's Gynecological Pathology of the Female Genital Tract. Kurman RJ (ed). 6th edition. Springer, New York, pp987-990, 2002.

6. Halperin R, Zehavi S, Hadas E, et al: Simultaneous carcinoma of the endometrium and ovary vs endometrial carcinoma with ovarian metastases: a clinical and immunohistochemical determination. Int J Gynecol Cancer 13: 32-37, 2003.

7. Soliman PT, Slomovitz BM, Broaddus RR, et al: Synchronous primary cancers of the endometrium and ovary, a single institution review of 84 cases. Gynecol Oncol 94: 456-462, 2004.

8. Nishimura N, Hachisuga T, Yokoyama M, et al: Clinicopathologic analysis of the prognostic factors in women with coexistence of endometrioid adenocarcinoma in the endometrium and ovary. J Obstet Gynaecol Res 31: 120-126, 2005.

9. Chiang YC, Chen CA, Huang CY, et al: Synchronous primary cancers of the endometrium and ovary. Int J Gynecol Cancer 18: 159-164, 2008.

10. Signorelli M, Fruscio R, Lissoni AA, et al: Synchronous earlystage endometrial and ovarian cancer. Int J Gynaecol Obstet 102: 34-38, 2008.

11. Eifel PJ, Ross J, Hendrickson M, Cox RS, Kempson R and Martinez A: Adenocarcinoma of the endometrium. Analysis of 256 cases with disease limited to the uterine corpus: treatment comparisons. Cancer 52: 1026-1031, 1983. 